\title{
Cytoskeletal changes in erythrocytes during storage in banked blood
}

\author{
Muhammad Haris ${ }^{1}$, Najma Baseer ${ }^{2}$, Yasar Mehmood Yousafzai ${ }^{3}$, Sobia Haris ${ }^{4}$, Usman Naeem ${ }^{5}$, \\ Rabail $^{1}$, Farah Deeba ${ }^{4}$, Muhammad Jehangir Khan ${ }^{6}$
}

\section{Submitted: \\ October 12, 2020 \\ Accepted: \\ December 8, 2020}

\section{ABSTRACT}

Introduction: Erythrocytes have flexible, non-nucleated bi-concave shape with lipid bilayer cytoskeleton. Any alterations of erythrocyte shape make it susceptible to hemolysis. Blood for transfusion purpose is routinely stored in Citrate Phosphate Dextrose Adenine (CPDA-1) containing blood bags. During storage, blood undergoes an array of different morphological changes termed as "storage lesions" which makes it more fragile. This study was aimed to determine the structural and functional modifications in erythrocytes in CPDA-1 blood stored in local blood bank of KPK.

Material \& Methods: Blood from twenty healthy volunteer donors was taken and kept in CPDA-1 containing blood bags at Institute of Basic Medical Sciences (IBMS), Khyber Medical University (KMU). Hb-levels and Erythrocyte, Reticulocyte counts, Mechanical Fragility Index (MFI) and immunofluorescence staining for ankiyrin 1 protein were performed on fresh blood samples. Samples for reticulocyte count was taken for 5 consecutive days while for the remaining parameters, blood was taken at 5 days interval till day 20th. The light and fluorescence micrographs were obtained accordingly and osmotic fragility tests were performed.

Results: A significant mean reduction in erythrocyte counts and Hb-level was observed from day 0 to 20 ( $\mathrm{p}=0.001)$, while MFI increased from day 0 to day $20(12.88 \% \pm 7.58 \mathrm{p}=0.001)$. Reticulocyte count also decreased from day 0 up to day $5(\mathrm{p}=0.001)$. A weak association was observed between changes in MFI and erythrocyte morphology from day 0 to $20(\mathrm{r}=0.349)$, while the intensity and pattern of ankyrin 1 protein expression appeared to change from day 10.

Conclusion: Blood stored for a week has same properties as fresh blood, however, important structural alterations start to appear after the first week of storage and worsen with time. Therefore, to gain better transfusion results, blood stored for up to one week can safely be transfused.

Key Words: Ankyrin1 protein, Blood storage, Cytoskeleton, Erythrocytes storage, Fluorescence microscope, Mechanical fragility index, Reticulocyte count, Transfusion safety.

The authors declared no conflict of interest and agreed to be accountable for all aspects of the work in ensuring that questions related to the accuracy or integrity of any part of the work are appropriately investigated and resolved.All authors contributed substantially to the planning of research, questionnaire design, data collection, data analysis and write-up of the article.

This article may be cited as:Haris M, Baseer N, Yousafzai YM, Haris S, Naeem U, Rabail, Deeba F, Khan MJ. Cytoskeletal changes in erythrocytes during storage in banked blood. Reh J Health Sci. 2020;2(2). 64-71

\section{INTRODUCTION}

Erythrocytes are the erythroid lineage of these colony forming units. Erythrocytes endure five developmental stages between proerythroblast and mature erythrocyte in about one week during circulation with the help of erythropoietin. ${ }^{1}$ The first phase is called the pro-erythroblast phase, the second basophilic phase, the third phase is of poly chromatophilic erythroblastin, the fourth phase is of orthochromatophilic erythroblast, the fifth and last phase is the transformation to reticulocytes phase in which the cell disgorges its nucleus. It ranges from $0.5 \%$ up to $1.5 \%$ of erythrocytes in normal healthy adults. Reticulocytes get matured into erythrocytes when they enter the blood stream. ${ }^{2}$ In humans, the mature erythrocyte has a life cycle of about 120 days. ${ }^{3}$ The normal average number of erythrocytes in blood in an adult female and male is about 4.1 to $5.1 \times 106 / \mu \mathrm{l}$ and 4.5 to $5.9 \times 106 / \mu \mathrm{l}$ respectively. ${ }^{4}$ The erythrocytes have a stretchy biconcave disc appearance, which allows them to go effortlessly through the minute vessels. The bi-concave character of the erythrocyte upsurges its surface area making the gaseous exchange to take place easily. The cytoskeleton of erythrocytes is composed of lipid bilayer with a range of cytoskeletal proteins suspended from it. The cytoskeleton of erythrocytes is composed of cytoskeletal proteins suspended from lipid bilayer. Underneath the lipid bilayer membrane is present a spectrin-actin cytoskeleton, which is united by junctional complex that forms aplain hexagonal structure. ${ }^{5}$ The basic framework of the erythrocyte membrane is composed of a protein meshwork that armors the inner membrane surface. The membrane cytoskeleton comprises predominantly of spectrin, actin and its associated proteins (tropomyosin, tropomodulin, adducin, and dematin), protein 4.1R, and ankyrin. The integral protein that is responsible for the preservation of elastic nature and biconcave discoid structure of an erythrocyte is the Spectrin. The first connection location comprises spectrin-glycophorin C Bridge. The second, main connection site is the spectrin-ankyrin-band 3 bridge, liable for the deformability and constancy of erythrocytes. ${ }^{8}$ Ankyrin1 or erythroid ankyrin, also acknowledged as ANK1, is a protein that in humans is encrypted by the ANK1 gene. ${ }^{6,9}$ It is a type of anchoring protein found in erythrocyte membrane cytoskeleton. During routine blood storage, these proteins derange with aging and result in deformity of erythrocytes shape and size 
thus making them more susceptible to hemolysis. ${ }^{8,9}$ This characteristic is significant owing to variability in routine blood storage practices for transfusion purposes.

Blood transfusion has always been considered vital in any health system. Transfusion of the erythrocytes have lifesaving value in case of severe blood loss due to any trauma or injury, anemia, surgery, cancerous conditions, hemorrhage or any blood dyscrasias. ${ }^{4,6}$ Transfusion of whole blood can ease health and protect life if used applicably. Rendering to World Health Organization (WHO), ${ }^{10}$ proper usage of blood items is defined as "the transfusion of safe blood products only to treat a condition leading to significant morbidity or mortality that cannot be prevented or managed effectively by other means". ${ }^{11}$ Allogenic Blood (AB) transfusion is a key component of rehabilitation for patients with severe anemia, with more than $50 \%$ of patients admitted to intensive care units getting blood transfusions. ${ }^{12}$ Latest researches have proposed that the transfusion of stored blood is related with amplified mortality, diseased problems, and organ letdown after surgery and austere traumatic injury. ${ }^{13-15}$ Together the volume and the time of transfused blood products have been recognized as sovereign threat features for multisystem organ letdown. ${ }^{13-16}$ Often, blood transfusions are associated with increased postoperative morbidity. ${ }^{17,18}$ Blood is a valuable and vital resource and it should be cautiously managed and used only when truly needed. ${ }^{19-21}$ Regardless of the advancement in the wellbeing of allogeneic blood $(\mathrm{AB})$ transfusions, there are still some probable hazards such as immunological responses, transfusion-transmitted infection, and bacterial infection. ${ }^{22,23}$ Packed erythrocytes transfusion is extensively used for the management of patients with anemia of dissimilar etiologies. It is therefore significant that the transfused erythrocytes preserve their metabolic capability and perfunctory properties.

The Food and Drug Administration ( FDA ) approved whole blood collection and storage guidelines allows erythrocytes to be stored for up to 42 days, while the average storage time of transfused blood in the United States (US) is 15 days. This is because the blood transfusion after this period of storage leads to significant morbidity and mortality due to alterations in erythrocyte shape and structure, which are collectively termed as "storage lesions". There is a loss of surface to volume ratio, thus causing increase in mean corpuscular hemoglobin concentration ( $\mathrm{MCHC}$ ), osmotic fragility (OF), mechanical fragility (MF) and decrease in reticulocyte count. Such changes decrease deformability of erythrocytes. Stored blood cells undergo massive structural and functional changes during storage that may decrease the usefulness and viability of erythrocytes after transfusion due to altered fragility. ${ }^{24}$ Erythrocyte mechanical fragility (MF) refers to the tendency of erythrocytes to hemolyze or rupture due to shear stress. Whereas, the mechanical fragility index (MFI) can be assumed as the percentage of hemolysis that ensues when a sample of erythrocyte are exposed to mechanical and shear stress. This suggests that when cells undergo different levels of stress, a fragility profile can be computed by assessing the comparative or absolute extent of hemolysis prevailing at each level. ${ }^{25,26}$ The increase in $\mathrm{OF}$ is suggestive of storage dependent membrane instability. ${ }^{27}$ Hence, the mechanical properties of erythrocytes are primarily determined by cell shape, surface area to volume ratio, surface charge, membrane and cytoskeleton.
Cross linkages when gets genetically disrupted, result in a congenital hemolytic anemia i-e; hereditary spherocytosis (HS) in which normal biconcave disc shape of erythrocytes is distorted and sphere-shaped cells are formed. ${ }^{10,12}$ Similarly, Glucose-6phosphate dehydrogenase (G6PD) deficiency is also a common human genetic disorder associated with changes in glutathione redox status or band 3 cytoskeleton complex interactions. ${ }^{12}$ Therefore, the objective of erythrocyte storage is to maintain its function and viability during the approved storage period. But modern storage mediums alter the functionality and viability of erythrocytes progressively through increased oxidative stress, altered metabolism and membrane damage. ${ }^{13}$ The survival time of erythrocytes after 24 hours of transfusion is inversely proportional to the length of storage period. Such changes cause higher mortality rate, serious infections and multi organ failure in indoor patients. ${ }^{14}$ Hence, the determination of time-dependent changes may be useful in better transfusion practices and reduced post transfusion mortality and morbidity.

Erythrocytes stored for transfusion purposes are prone to an array of morphological changes. In contrast to the aforementioned studies, the morphological changes have been identified using light microscope and scanning electron microscope (SEM). However, not much work has been done to determine the time-dependent cytoskeletal changes in erythrocytes structural proteins i-e ankyrin 1 using immunofluorescence in banked blood. Similarly, the time-dependent changes in reticulocyte count as well as mechanical fragility of the erythrocytes in banked blood has not been studied much. So, this study was set to determine the effect of storage on the erythrocyte cytoskeleton in banked blood. This will be helpful in understanding the pathogenesis of erythrocytes structural disorders, thus devising ways for better diagnosis, prognosis and cure.

\section{MATERIAL \& METHODS}

This was a laboratory based experimental study, which was executed in six months after evaluation and endorsement by Graduate Studies Committee and subsequent approval by the Advanced Studies and Research and ethics Board of KMU (DIR/KMUAS\&RB/CC/000840, and Ethical Review Board vide No: Dir/KMUEB/CC/000593) and Fatimid Foundation, Peshawar. Twenty healthy volunteer of age 17 to 40 years based on Erik Erikson's classification of young adult ${ }^{25}$ blood donors' blood was taken in CPDA-1 containing blood bags at Fatimid Foundation, Peshawar. The donors' blood collected by Fatimid Foundation staff was then screened for Hepatitis B, Hepatitis C and HIV by Elisa method. People who were anemic clinically having pale conjunctivae, Hepatitis B, Hepatitis C and HIV positive and those not fit according to WHO standard guidelines for blood donation were excluded from this study. ${ }^{25}$

After the blood was screened for the aforementioned infections, it was then processed in centrifuge (Crifuge) at $4^{\circ} \mathrm{C}$ for 8 minutes at $2800 \mathrm{rpm}$ to separate erythrocytes. All the bags of packed erythrocytes were then stored in the blood bank of Fatimid Foundation at +2 to $+6^{\circ} \mathrm{C}$ for about 20 days. The initial blood sample was collected within first four hours to observe reticulocyte count, Mechanical Fragility Index, Giemsa staining, mechanical fragility index of the erythrocytes and Immunofluorescence. Before collection of samples, the packed erythrocytes bags were variegated 
This was a laboratory based experimental study, which was executed in six months after evaluation and endorsement by Graduate Studies Committee and subsequent approval by the Advanced Studies and Research and ethics Board of KMU (DIR/KMU AS\&RB/CC/000840, and Ethical Review Board vide No: Dir/KMUEB/CC/000593) and Fatimid Foundation, Peshawar. Twenty healthy volunteer of age 17 to 40 years based on Erik Erikson's classification of young adult ${ }^{25}$ blood donors' blood was taken in CPDA-1 containing blood bags at Fatimid Foundation, Peshawar. The donors' blood collected by Fatimid Foundation staff was then screened for Hepatitis B, Hepatitis C and HIV by Elisa method. People who were anemic clinically having pale conjunctivae, Hepatitis B, Hepatitis C and HIV positive and those not fit according to WHO standard guidelines for blood donation were excluded from this study. ${ }^{25}$

After the blood was screened for the aforementioned infections, it was then processed in centrifuge (Crifuge) at $4^{\circ} \mathrm{C}$ for 8 minutes at $2800 \mathrm{rpm}$ to separate erythrocytes. All the bags of packed erythrocytes were then stored in the blood bank of Fatimid Foundation at +2 to $+6^{\circ} \mathrm{C}$ for about 20 days. The initial blood sample was collected within first four hours to observe reticulocyte count, Mechanical Fragility Index, Giemsa staining, mechanical fragility index of the erythrocytes and Immunofluorescence. Before collection of samples, the packed erythrocytes bags were variegated by mechanical tray to avoid blood clotting. Successive blood samples were collected at 5 days interval on $5^{\text {th }}$ Day, $10^{\text {th }}$ Day, $15^{\text {th }}$ Day and last sample was collected at $20^{\text {th }}$ Day of erythrocyte storage period. Packed erythrocytes sample of about $5 \mathrm{ml}$ was collected in $5 \mathrm{ml}$ syringe properly labeled from each pre-labeled blood bags on the same day within 4 hours of blood collection, $5^{\text {th }}$ Day, $10^{\text {th }}$ Day, $15^{\text {th }}$ Day and $20^{\text {th }}$ Day for the parameters mentioned above. Reticulocyte staining for Reticulocyte count was done on daily basis from 0 day up to 5th Day. Pilot test was run to optimize and validate the laboratory protocols for mechanical fragility index and immunofluorescence of staining of ankyrin 1 antigen. For the remaining parameters such as reticulocyte count and Giemsa staining, validated protocols were used.

For mechanical fragility, approximately $1.5 \mathrm{ml}$ erythrocyte concentrate taken in three $4 \mathrm{ml}$ plain glass tubes labelled as; $\mathrm{BB}$ (containing 1 Steel Ball Bead), PC (Positive Control), NC (Negative Control). $2 \mathrm{ml}$ of $0.9 \%$ normal saline added to each tube. Tube BB and $\mathrm{PC}$ were rocked at $40 \mathrm{cycles} / \mathrm{min}$ at $45^{\circ}$ for $15 \mathrm{~min}$ at room temp $(22 \pm$ $1^{\circ} \mathrm{C}$ ) in mixer. Afterwards, all the three tubes were centrifuged at $3000 \mathrm{rpm}$ for 3 minutes at room temp. The supernatant in each tube was collected in three cuvettes labeled as BB, PC and NC. Spectrophotometer balanced to zero (by measuring optical density (OD) of $0.9 \%$ normal saline at $546 \mathrm{~nm}$ ). OD checked for free $\mathrm{Hb}$ in the supernatant collected in each tube $\mathrm{BB}, \mathrm{PC}$ and $\mathrm{NC}^{26-28}$ The hemolysis level (\% hemolysis or MFI) was calculated according to the formula; $\mathrm{MFI}=100 \times\left(\mathrm{OD}_{\mathrm{BB}}-\mathrm{OD}_{\mathrm{C}}\right) /\left(\mathrm{OD}_{\mathrm{T}}-\mathrm{OD}_{\mathrm{C}}\right)$, whereas, the subscript $\mathrm{C}, \mathrm{BB}$, and $\mathrm{T}$ corresponds to the $\mathrm{OD}$ of supernatant obtained from Control, Ball bead and Total hemolysis group respectively. ${ }^{26}$ The supernatant collected from each plain glass tube was subjected to measurements of hemolysis level providing MFI (\% of hemolysis of Erythrocytes subjected to mechanical stress). ${ }^{28}$

Immunofluorescence staining was performed in order to visualize the changes taking place in the cytoskeletal proteins of the erythrocytes using primary and secondary antibody complex against Ankyrin1 antigen. 50 $\mu \mathrm{l}$ of erythrocyte concentrate was diluted in $200 \mu \mathrm{l}$ of $0.9 \%$ sodium chloride in a $1.5 \mu$ l cuvette. Cells were fixed using $100 \mu \mathrm{l}$ of $4 \%$ formalin which was added to the pre-diluted mixture of erythrocytes. Afterwards, smear was made on coated slides and then air dried. Smear was washed with PBS and then Peroxidase blocking reagent was applied for about $5 \mathrm{mins}$. Smear was again washed 3 times with PBS for about 2 mins each. Monoclonal mouse anti-human ankyrin1 primary antibody was applied for overnight at $4^{\circ} \mathrm{C}$. Smear was again washed 3 times with PBS for about 2 mins each. Goat anti-mouse secondary antibody (488) was applied for aboutts 1 hour at room temperature. Smear was again washed 2

This study was aimed to assess the structural modifications in erythrocytes during storage period. The optimal results for MFI were observed with the formula $\mathrm{MFI}=100 \times\left(\mathrm{OD}_{\mathrm{BB}}-\mathrm{OD}_{\mathrm{C}}\right) /\left(\mathrm{OD}_{\mathrm{T}}-\mathrm{OD}_{\mathrm{C}}\right)$, whereas the subscript $\mathrm{C}, \mathrm{BB}$, and $\mathrm{T}$ corresponds to the $\mathrm{OD}$ of supernatant obtained from Control, Ball bead and Total hemolysis group respectively. ${ }^{32,33}$ During the pilot study, a rise in MFI was observed with MFI on day 0 as $13.34 \% \pm 6.28$ (Mean \pm S.D) to $27.78 \% \pm 5.31($ Mean \pm S.D) on day 20 of the storage period.

For the optimization of antibody concentration, multiple concentrations of primary and secondary antibodies were used for erythrocyte staining i.e., 1:50, 1:100, 1:300, 1:500 for (Mouse antihuman Ankyrin1 primary antibody) whereas 1:100 and 1:500 for (Alexa Fluor 488 Goat anti-mouse secondary antibody). But the staining results were optimum with 1:300 (Mouse anti-human Ankyrin1 primary) and 1:100 (Alexa Fluor 488 Goat anti-mouse secondary) antibodies.

The average age of volunteer donors was $25.85 \pm 5.77$ years. All the donors were donating blood for the very first time. The subsequent blood profile was observed on day 0 and a conclusive baseline was drawn on the basis of changes that would occur on day 5 , day 10 , day 15 and finally on day 20 whereas keeping the readings of day 0 as control. For more substantial results, the readings from day 5 and day 10 were further grouped as one group (Group A) whereas day 15 and day 20 were grouped as second group (Group B). Readings were observed and compared for the following parameters;

Time-dependent changes in;

Hematological Parameters of banked erythrocytes.

Reticulocyte count of banked erythrocytes.

MFI and its correlation with changes in morphology of banked erythrocytes.

Ankyrin 1 protein expression in banked erythrocytes.

\section{Hematological Parameters of banked erythrocytes:}

The time-dependent changes in hematological parameters i.e., Erythrocyte count and Hb-level of banked erythrocytes were checked by means of hematological analyzer BC 3000 (Mindray, Schenzhen China). The hematological parameters in twenty healthy donors packed erythrocytes at five days interval i.e.; $0,5^{\text {th }}, 10^{\text {th }}, 15^{\text {th }}$ and $20^{\text {th }}$ day demonstrated a steady decrease in mean erythrocyte count and $\mathrm{Hb}$-levels. The mean erythrocytes count was $5.27 \times 10^{12} / \mathrm{L} \pm 0.37$ on day 0 , whereas on day 20 , it got decreased to $4.48 \times 10^{12} / \mathrm{L} \pm 0.29$. The mean decrease in erythrocyte counts from day 0 to $20^{\text {th }}$ was $0.79 \times 10^{12} / \mathrm{L} \pm 0.08$. For inferential statistics, One-way ANOVA test was done. The $p$ value for ANOVA was significant ( $p=0.001)$. During storage period, the Hb-level got reduced in relation to time. At 0 day, the average $\mathrm{Hb}$-level was noted as 
$14.45 \mathrm{~g} / \mathrm{dl} \pm 1.25$, whereas on $20^{\text {th }} \mathrm{day}$, it got reduced to $12.85 \mathrm{~g} / \mathrm{dl} \pm 0.65$. The mean decrease in $\mathrm{Hb}$-level from day 0 to $20^{\text {th }}$ was $1.60 \mathrm{~g} / \mathrm{dl} \pm 0.60$.

\section{Reticulocyte count of banked erythrocytes:}

During storage period, the reticulocyte count decreased slightly from day 0 to day 5 . The mean reticulocyte count on day 0 was $0.69 \% \pm 0.16$ whereas on day 5 , it was $0.475 \% \pm 0.138$. The averag $\epsilon$ decrease in Reticulocyte count from day 0 up to day 5 was $0.215 \% \pm 0.022(p=0.000$ at $\leq 0.05)$. For multiple comparisons, Pos 1 Hoc Tukey test was done which illustrated a significant difference ir Reticulocyte count of day 0 , day 1 and day 2 with day 5 . Whereas there was no significant difference in Reticulocyte count on days 3, 4 and 5. In addition, there was a substantial drop in Reticulocyte count over the period of time from day 0 up to day 5 of storage perioc (Figure $1 \& 2$ ).

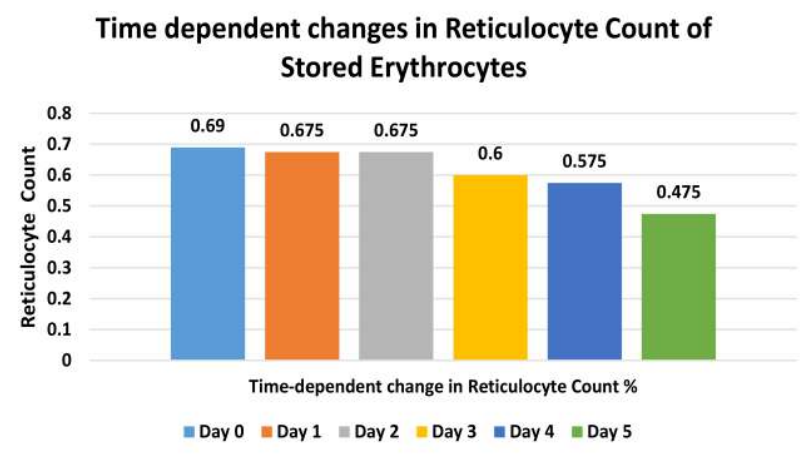

Figure 1: Graph showing Time-dependent change in Reticulocyte count of stored erythrocytes.
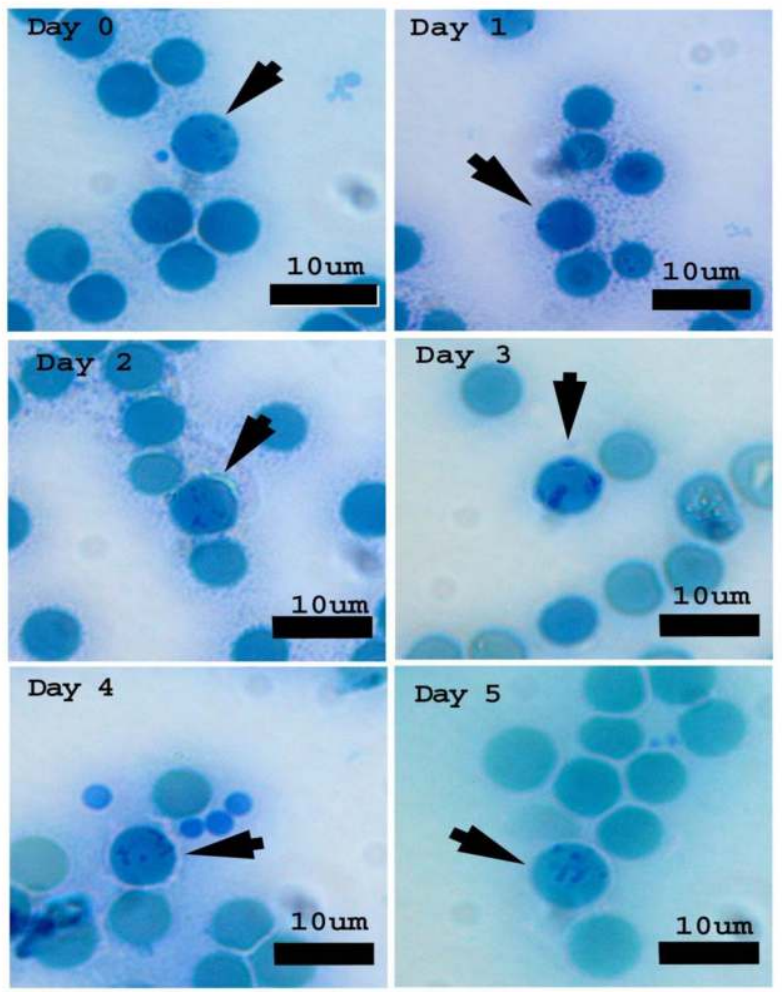

Figure 2: Light micrographs showing Reticulocytes of Day 0, 1,2,3,4 and Day 5 stained with new methylene blue. The arrow heads are showing stained reticulocytes.
Time-dependent changes in Mechanical Fragility Index and its correlation with changes in Morphology of banked Erythrocytes: The MFI increased from day 0 to day 20. The average MFI on day 0 was $19.99 \% \pm 13.20$, whereas on day 20 , it increased up to $32.87 \% \pm 20.78$. The mean increase in MFI from day 0 up to day 20 was $12.88 \% \pm 7.58$ as shown in figure 3 .

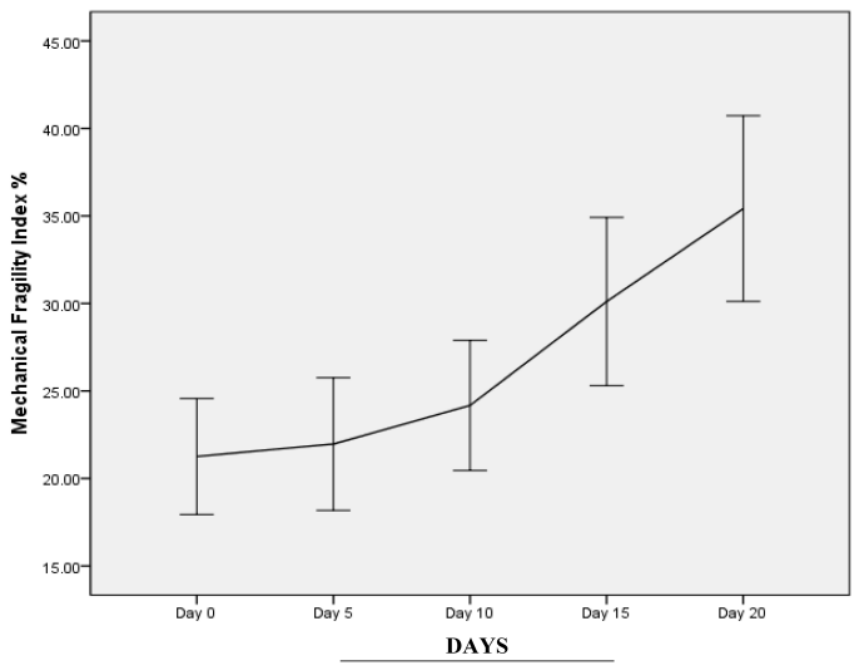

Figure 3: Changes in MFI with relation to change storage period.

In order to calculate the mean increase in MFI at different time points, the samples were grouped into four groups i.e. Group A (Day 0-5), Group B (Day 0-10), Group C (Day 0-15) and Group D (day 0-20). In these groups, the percentage increase in MFI was calculated by subtracting MFI on day 0 from the MFI on Day 5 , 10, 15 and 20. One-way ANOVA test was done and the result of all 4 groups were statistically significant $(\mathrm{p}=0.001)$. For Post Hoc analysis, Tukey test was performed, which showed that among these groups, Group A and Group B were different from Group D $(\mathrm{p}=0.008)$. Moreover, Group $\mathrm{C}$ was not significantly different from Group D, while Group C and Group D were also not significantly different from each other.

Similarly, changes in erythrocyte morphology with increasing percentage of abnormal erythrocytes with time during storage period were also determined (Figure 4). Furthermore, the percentage increase in abnormal erythrocytes with time was computed with respect to day 0,10 and 20 and the mean difference between the groups was analyzed using One-way ANOVA, which showed significant results $(\mathrm{p}=0.000)$. Moreover, Post Hoc Tukey test also showed significant difference between these groups $(\mathrm{p}=0.000)$. In order to determine whether there was any association between the percentage increase in MFI and abnormal erythrocytes at days 0,10 and 20 , correlation statistics were also performed. For this, the data was grouped as Group A (Day 0-10) and Group B (Day 0-20) in which the MFI and percent change in abnormal erythrocytes on day 0 were subtracted from that of day 10 and day 20 respectively. There was a weak but significant positive correlation ( $\mathrm{r}=0.35)$ at $95 \%$ confidence level between increase in MFI and abnormal erythrocytes with respect to time points at day 0,10 and 20 . 

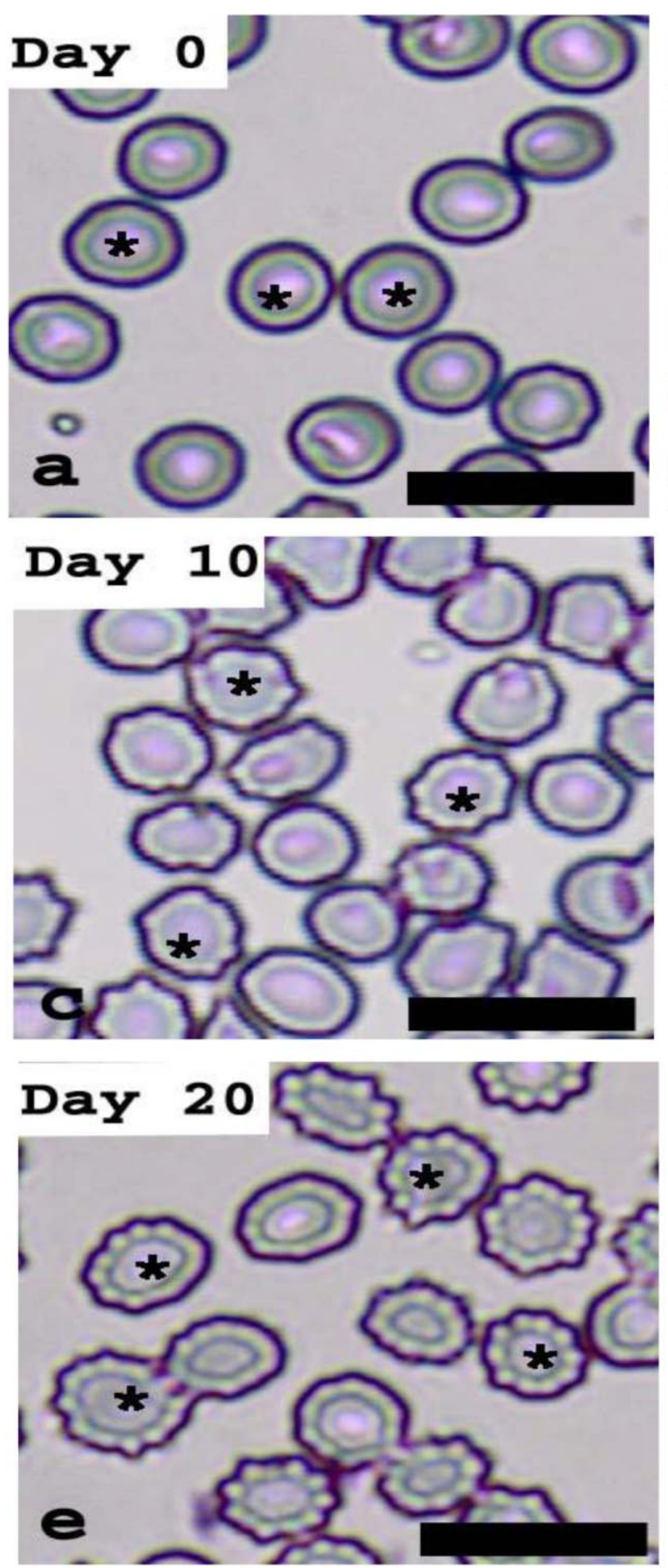
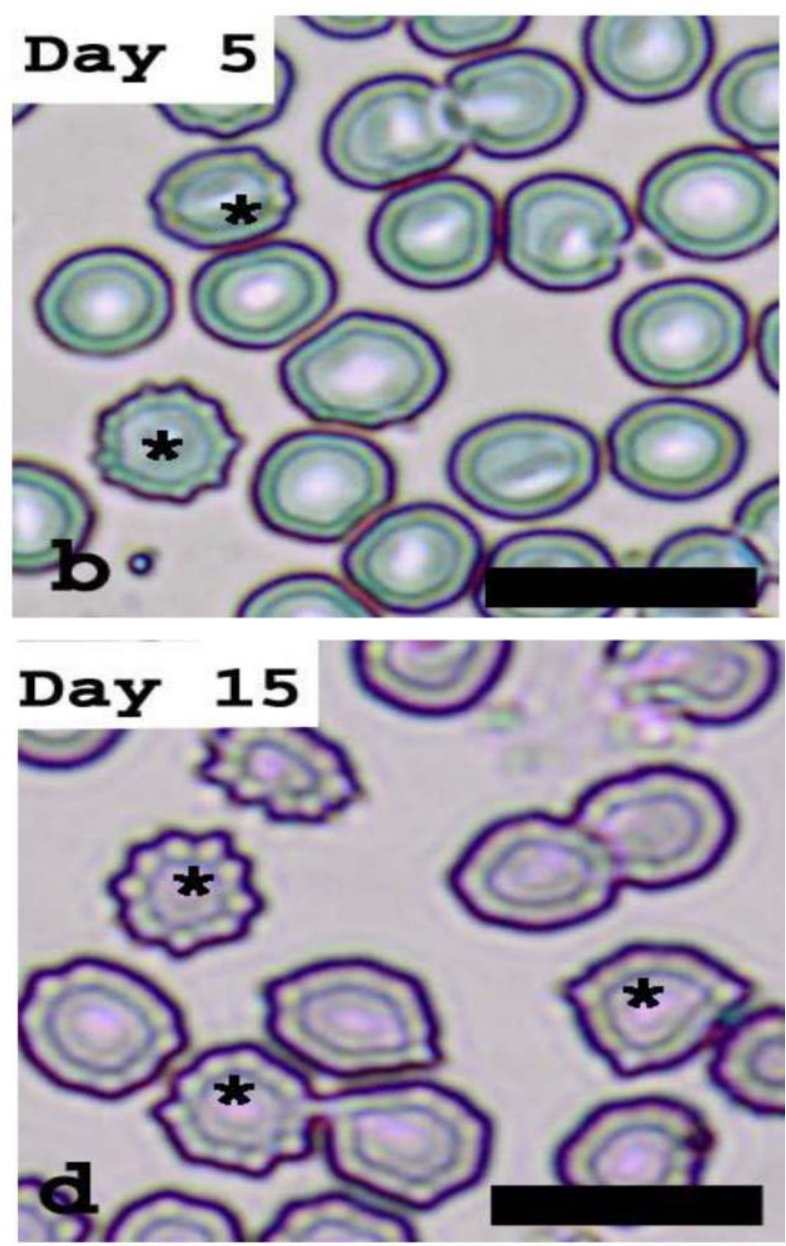

Figure 4: Light micrographs with scale bar of $10 \mu \mathrm{m}$ showing erythrocytes of day 0 , day 5 , day 10 , day 15 and day 20 of changed morphology from normal bi-concave discoid morphology stained with Giemsa stain. In Figure: 4(a), the asterisk is showing normal erythrocytes whereas in Figure: 4(b), 4 C, 4(D) and 4 (E), and it is showing altered (Spherocytes) morphology of Erythrocytes.
Time-Dependent Changes in Ankyrin-1 Protein Expression in Banked Erythrocytes

Immunofluorescence staining was done for ankyrin1 protein with monoclonal mouse anti-human ankyrin 1 primary antibody, which was subsequently immuno-stained with Alexa Fluor 488 goat antimouse secondary antibody. On day 0 , there was a complete rim and uniform staining pattern of erythrocytes outer membrane Figure 5(a). Normal discoid and bi-concave shape was observed with sharp staining intensity as shown below in Figure 5(a). On day 5, the staining intensity got reduced. Incomplete rims were observed. However, normal bi-concave and discoid shape still dominated the field as shown below in Figure 5(b). Interestingly, on day 10, there was punctate staining pattern as compared to uniform staining pattern observed on day 0 and 5. Moreover, the erythrocytes apparently shrunk and normal bi-concave discoid shape also appeared to be disrupted and the staining intensity was also reduced, as shown below in Figure 8(c). Unfortunately, despite repeating the same staining protocols about six times, no immunofluorescence 

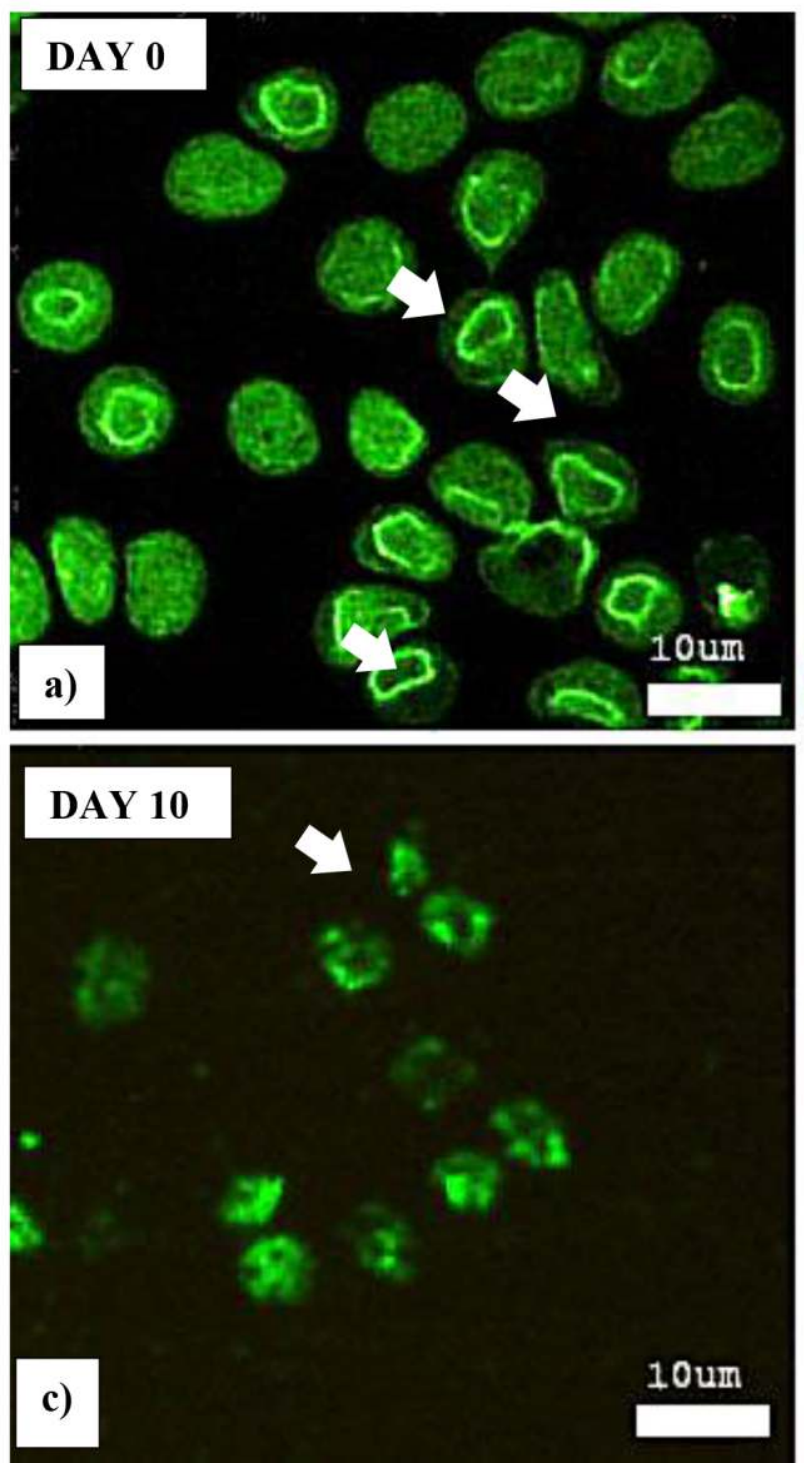

\section{DISCUSSION}

The foremost purpose of this research was to explore the alterations that occur in the reticulocyte count and in the morphology of erythrocytes stored in the banked blood over the period of time. Moreover, the vulnerability of erythrocytes to mechanical stress was also explored using mechanical fragility index test. In all the twenty healthy volunteer donors' blood, the hematological parameters i.e., erythrocyte count, $\mathrm{Hb}$ levels and mechanical fragility index were checked with 5 days interval. The reticulocyte count and changes in the morphology of erythrocyte were also assessed using multiheaded light microscope and fluorescence microscope.

Time-Dependent Changes in Hematological Parameters of Banked Erythrocytes:

A substantial decrease in erythrocyte count and Hb-levels were noted. The decrease in Hb-levels started as promptly as on day 5 . The probable justification to this decrease is the breakdown of senescent erythrocytes in stored packed erythrocytes. The aforementioned results are in line with Tuo, W., W., et al. ${ }^{33}$ who stored whole blood in blood bag containing CPDA-1 solution and detected the alterations in hematological parameters with seven days interval. The changes in

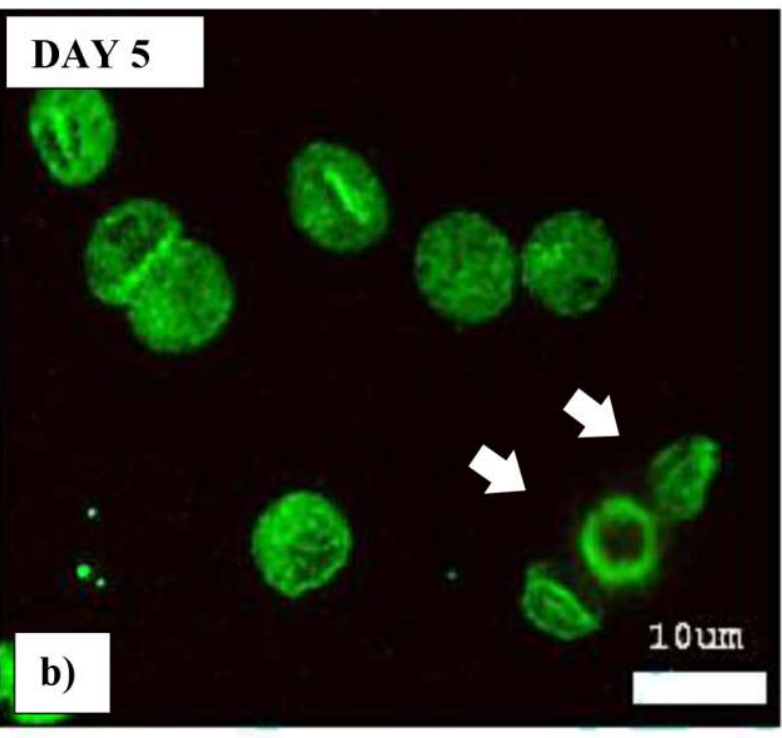

Figure 5: Fluorescent micrograph showing time-dependent changes in Ankyrin1 protein expression in banked Erythrocytes. On Day 0, complete rim of erythrocytes outer membrane and normal morphology is visible (arrows). On day 5 , some distorted cells are visible while the outer membrane is still intact in the remaining normal cells (arrows). On day 10, both the morphology and staining pattern of Ankyrin seems disrupted (arrows). Magnification $=40 x$, Scale bar 10um such parameters were not significant on day 0 , whereas on day 7 , there was substantial increase in plasma Hb-levels. Likewise, separating erythrocytes from whole blood using density difference, they also revealed that the numerous senescent erythrocytes abruptly rises after 14 days of storage period. Collectively, these outcomes were suggestive of erythrocyte speedy rupture, which resulted in reduced overall Hb-levels and erythrocyte counts.

Time-Dependent Changes in Reticulocytes Count of Banked Erythrocytes:

A significant decrease in reticulocytes count was observed over the period of time from day 0 up to day 5 . Ashild et al. ${ }^{34}$ showed a

slight increase in the reticulocyte count of thalassemia patients blood stored for up to six days. However, this may be due to increased hemolysis of erythrocytes in such patients' blood. Such increased hemolysis falsely increases reticulocyte count whereas the actual reticulocyte count slightly decreases over the period of time in healthy stored blood. As stated in this study, the aforementioned results in previous studies are in line with the current study. In other study of Urbina A. et al..$^{35}$, there was a marked decline in mean reticulocyte count of erythrocytes being 
The foremost purpose of this research was to explore the alterations that occur in the reticulocyte count and in the morphology of erythrocytes stored in the banked blood over the period of time. Moreover, the vulnerability of erythrocytes to mechanical stress was also explored using mechanical fragility index test. In all the twenty healthy volunteer donors' blood, the hematological parameters i.e., erythrocyte count, $\mathrm{Hb}$ levels and mechanical fragility index were checked with 5 days interval. The reticulocyte count and changes in the morphology of erythrocyte were also assessed using multiheaded light microscope and fluorescence microscope.

Time-Dependent Changes in Hematological Parameters of Banked Erythrocytes:

A substantial decrease in erythrocyte count and Hb-levels were noted. The decrease in Hb-levels started as promptly as on day 5 . The probable justification to this decrease is the breakdown of senescent erythrocytes in stored packed erythrocytes. The aforementioned results are in line with Tuo, W., W., et al. ${ }^{33}$ who stored whole blood in blood bag containing CPDA-1 solution and detected the alterations in hematological parameters with seven days interval. The changes in such parameters were not significant on day 0 , whereas on day 7 , there was substantial increase in plasma Hb-levels. Likewise, separating erythrocytes from whole blood using density difference, they also revealed that the numerous senescent erythrocytes abruptly rises after 14 days of storage period. Collectively, these outcomes were suggestive of erythrocyte speedy rupture, which resulted in reduced overall $\mathrm{Hb}$-levels and erythrocyte counts.

Time-Dependent Changes in Reticulocytes Count of Banked Erythrocytes:

A significant decrease in reticulocytes count was observed over the period of time from day 0 up to day 5 . Ashild et al. ${ }^{34}$ showed a slight increase in the reticulocyte count of thalassemia patients' blood stored for up to six days. However, this may be due to increased hemolysis of erythrocytes in such patients' blood. Such increased hemolysis falsely increases reticulocyte count whereas the actual reticulocyte count slightly decreases over the period of time in healthy stored blood. As stated in this study, the aforementioned results in previous studies are in line with the current study. In other study of Urbina A. et al. ${ }^{35}$, there was a marked decline in mean reticulocyte count of erythrocytes being stored in AS-1 solution during the entire storage period. Such decline is suggestive of that these reticulocytes get matured during storage period. Similarly, Lippi G. et al. ${ }^{36}$ demonstrated that there are no significant changes in the reticulocytes count of blood sample collected within 4-hours of storage period after collection from donor and that of day 1 , which is again consistent with the results of the current study. Keeping in view the aforementioned studies, it is suggestive that reticulocytes counts decrease as the storage period progresses because of reticulocytes maturation which are in accordance with the findings of our study.

Time-Dependent Changes in Mechanical Fragility Index and Its Correlation with Changes in Morphology of Banked

\section{Erythrocytes:}

A significant change in erythrocytes morphology was observed during the course of storage period when examined under multiheaded light microscope. The structural changes in the erythrocytes started after 5 th day of storage period. This was owing to the fact that the erythrocytes morphology alters because of cytoskeletal instability in their cell membrane during storage period. The results in this study are quite similar with the studies done in past. Mustafa I et al. ${ }^{37}$ presented the structural disparities in leuko-reduced packed erythrocytes stored for about 42 days in SAGM (Saline, Adenine, Glucose, Mannitol) medium. The structural alterations in erythrocytes were seen through scanning electron microscope on 0 day, 14 th day, 28th day and 42 nd day of storage period. Day 0 was selected as control. It depicted normal erythrocyte with normal biconcave disc morphology. Whereas, there were significant changes in normal bi-concave discoid shape of erythrocyte and more spherocytic cells were formed as the storage period progressed to 14 th day. Such changes in morphology of erythrocytes could have been depicted before 14th day as observed in the current study on 5th day through light microscope and fluorescence microscope. However, lengthy recess of 14 days ensued late changes. Furthermore, difference in blood banking protocols may also elucidate the dissimilarities between two researches. Park, H.et al. ${ }^{38}$ calculated the biophysical characteristics of banked erythrocytes by means of 3-D refractive tomograms (3-D RI), measured with common-path diffraction optical tomography(cDOT). Whole blood was stored in blood bags containing CPDA-1 and those without CPDA- 1 solution and studied on 0 day, 5th day, 13th day, 20th day, 27th day, 34th day and 41 st day. More than $60 \%$ of CPDA- 1 stored erythrocytes presented with a non-discocyte form at 5 th day. Whereas structural changes i.e., non-discocyte form of erythrocytes were significantly reduced to $20 \%$ in erythrocytes stored in CPDA-1 solution at 5 th day of storage period. Likewise, Blasi, B., D'alessandro et al. ${ }^{32}$ stored erythrocytes in SAGM solution and studied it weekly from 0 day up to 42 nd day of storage period. They observed the revocable and irrevocable morphological changes (i.e.; spherocytosis was markedly enhanced) in

\section{CONCLUSION}

This project was aimed at determining the hematological and structural alterations, when blood kept at $2{ }^{\circ} \mathrm{C}$ to $6^{\circ} \mathrm{C}$ for up to 20 days. The hematological factors observed were the erythrocyte counts and $\mathrm{Hb}$ concentration. Significant variations in erythrocyte counts and $\mathrm{Hb}$ concentration were noted. Similarly, substantial reduction in reticulocyte count was observed during storage period from day 0 up to day 5. Moreover, structural alterations were detected on 5th day of blood storage by use of multi-headed light microscope. The mechanical fragility amplified over period of time. These outcomes recommended that about seven days old blood can as safely be used as fresh blood. Though, important structural alterations begin to seem after the initial seven days of storage and such alterations get worsen as the storage period progresses. Therefore, to attain finest conceivable transfusion results, blood stored for up to seven days can safely be used for transfusion purpose. Furthermore, Advance researches on the outcome of transfusion of stored blood in healthy and unhealthy ones should be carried out to understand the clinical repercussions of transfusion practices. In addition, more cytoskeletal proteins should be explored using standard protocols i.e., Cytospin and other appropriate methods should be considered. A comprehensive assessment of blood storage situation of all the blood banks in Khyber Pakhtunkhwa as well as in the whole country should be done which will support in revising our storage strategies. 


\section{LIMITATIONS}

Our study also had certain constraints that might have affected the interpretation of our research findings to some extent. As far as the study impact is concerned, sample size was small. Due to short duration of the study and lack of predefined laboratory protocols, the great amount of time was consumed in establishing the optimal protocols for immunofluorescence staining. Due to which only a limited number of immunofluorescence reactions were carried out. Furthermore, due to financial constraints and high cost of antibodies, it was difficult to explore multiple cytoskeletal proteins.

\section{RECOMMENDATIONS}

In the response to the aforementioned constraints, the authors of the study suggest that more advanced researches should be carried on the outcome of transfusion of stored blood in healthy and unhealthy individuals to understand the clinical repercussions. Furthermore, due to intricacy of the erythrocytes cytoskeleton, more proteins should be explored using standard protocols and advanced equipment such as i.e., cytospin. Having a multicentric approach with a bigger sample size may yield an outcome with a more promising impact. Therefore, a comprehensive assessment of blood storage situation of all the blood banks in Khyber Pakhtunkhwa as well as in the whole country should be done which will assist in revising our blood storage guidelines.

\section{REFERENCES}

1) Adamson, J.W. Regulation of red blood cell production. The American journal of medicine 101.1996; 4S-6S.

2) Mescher, A. L. Junqueira's basic histology: Text and Atlas. McGraw Hill, 2013.

3) Franco, R. S. Measurement of red cell lifespan and aging. Transfusion medicine and hemotherapy 39. 2012; 302-307.

4) Chmielewski, P., Strzelec, B., Chmielowiec, J., Chmielowiec, K. \& Borysławski, K. Association between body size and selected hematological parameters in men and women aged 45 and above from a hospitalized population of older adults: an insight from the Polish Longitudinal Study of Aging (1960-2000). Anthropological review 80. 2017; 171-190.

5) Buys, A. V. et al. Changes in red blood cell membrane structure in type 2 diabetes: a scanning electron and atomic force microscopy study. Cardiovascular diabetology 12. 2013; 25.

6) Baskurt, O. K., Meiselman, H. J. in Seminars in thrombosis and hemostasis. Thieme Medical Publishers, Inc. 2003; 435-450.

7) Pesciotta EN, Sriswasdi S, Tang HY, Mason PJ, Bessler M, Speicher DW. A label-free proteome analysis strategy for identifying quantitative changes in erythrocyte membranes induced by red cell disorders. $J$ Proteomics. 2012; 76(Spec No):194-202.

8) Willardson, B., M., Thevenin, B.J., Harrison, M., L., Kuster, W., M., Benson, M., D. and Low, P., S., Localization of the ankyrin-binding site on erythrocyte membrane protein, band 3. J Biol Chem. 1989; 264(27):1589315899.

9) Lambert S, Yu H, Prchal JT, et al. cDNA sequence for human erythrocyte ankyrin. Proc. Natl. Acad. Sci. U.S.A. 1990; 87 (5): 1730-4.

10) World Health Organization. The clinical use of blood in obstetrics, pediatrics. Surgery \& Anaesthesia, Trauma \& Burns; 2001.

11) World Health Organization. Blood Transfusion Safety: Safe and Appropriate Use; 2002.

12) Corwin HL, Gettinger A, Pearl RG, Fink MP, Levy MM, Abraham E, MacIntyre NR, Shabot MM, Duh MS, Shapiro MJ. The CRIT Study: anemia and blood transfusion in the critically ill-current clinical practice in the United States. Crit Care Med. 2004; 32:39-52.

13) Koch CG, Li L, Sessler DI, Figueroa P, Hoeltge GA, Mihaljevic T, Blackstone EH. Duration of red-cell storage and complications after cardiac surgery. $\mathrm{N}$ Engl J Med. 2008; 358:1229-1239.

14) Offner PJ, Moore EE, Biffl WL, Johnson JL,
Silliman CC. Increased rate of infection associated with transfusion of old blood after severe injury. Arch Surg. 2002; 137:711-716.

15) Weinberg JA, McGwin G Jr, Griffin RL, Huynh VQ, Cherry SA 3rd, Marques MB, Reiff DA, Kerby JD, Rue LW 3rd. Age of transfused blood: an independent predictor of mortality despite universa leuko-reduction. J Trauma. 2008 ; 65:279-282.

16) Zallen G, Offner PJ, Moore EE, Blackwell J, Ciesla DJ, Gabriel J, Denny C, Silliman CC. Age of transfused blood is an independen risk factor for postinjury multiple organ failure. Am J Surg. 1999; 178:570-572.

17) Hill GE, Frawley WH, Griffith KE, et al. Allogeneic blood transfusion increases the risk of postoperative bacterial infection: a meta-analysis. JTrauma. 2003;54:908-14.

18) Fisahn C, Jeyamohan $S$, Norvell DC, et al. Association between allogeneic blood transfusion and postoperative infection in major spine surgery. Clin Spine Surg. 2017, 30:E 988-92.

19) Goodnough LT, Levy JH, Murphy MF. Concepts of blood transfusion in adults. Lancet. 2013; 381:1845-54.

20) Goodnough LT, Spahn DR. Alternatives to blood transfusion. Lancet. 2013 ; 381:1855-65.

21) Williamson LM, Devine DV. Challenges in the management of the blood supply. Lancet. 2013; 381:1866-75.

22) Shafiee A, Nazari S, Mogharreban M Koupaei MT. Evaluating medical interns' knowledge of common blood transfusion complications. Transfus Apher Sci. 2013 48(2):253-6.

23) Koch CG, Li L, Sessler DI, Figueroa $P$, Hoeltge GA, et al. Duration of red-cel storage and complications after cardiac surgery. N Engl J Med. 2008; 358: 1229 1239.http://spherocytosis.info/2012/01/os motic-fragility-test/

24) .Organization, W.H.

25) Barshtein G, Abramovitch R, Katz M, Pappo $\mathrm{O}$, Zelig $\mathrm{O}$, Corchia N, Yedgar S, Matot Aged versus fresh blood for the treatment of hemorrhagic shock; differential effect on liver outcome and possible mechanism. Vox Sang 2010; 99: 68.

26) Kaul DK, Koshkaryev A, Artmann G Barshtein G, Yedgar S: Additive effect of red blood cell rigidity and adherence to endothelial cells in inducing vascular resistance. Am J Physiol Heart Circ Physiol. 2008; 295.

27) Gregory Barshtein, Alexander Gural, Noga Manny, Orly Zelig, Saul Yedgara, Dan Arbell.
Storage Induced Damage to Red Blood Cell Mechanical Properties Can Be Only Partially Reversed by Rejuvenation. Transfus Med Hemother. 2014; 41:197-204.

28) Manual of Laboratory Medicine, 3rd Ed, 2005: 264.

29) Palmer, L. et al. ICSH recommendations for the standardization of nomenclature and grading of peripheral blood cell morphological features. International journal of laboratory hematology. 2015; 37 , 287-303.

30) Gregory Barshtein, Alexander Gural, Noga Manny, Orly Zelig, Saul Yedgara, Dan Arbell. Storage Induced Damage to Red Blood Cell Mechanical Properties Can Be Only $P$ a r t i I I y R e versed b y Rejuvenation.Transfus Med Hemother. 2014; 41:197-204.

31) Blasi B, D'Alessandro A, Ramundo $N$, Zolla L. Red blood cell storage and cell morphology. Transfus Med. 2012; 22:90-22.

32) Saleh, B. M. \& Bashi, A.Y. D. Effect of blood storage on certain hematological parameters. Medical Journal of Tikrit. 2009; 1,171-180.

33) Tuo, W.-W., Wang, D., Liang, W.-J., Huang, Y.$X$. How cell number and cellular properties of blood-banked red blood cells of different cell ages decline during storage. PloSone 9, e105692. 2014.

34) Åshild A. Sudmann-Day, Armin Piehler, Olav Klingenberg, Petter Urdal. Six-day stability of erythrocyte and reticulocyte parameters in-vitro: A comparison of blood samples from healthy, iron deficient and thalassemic individuals. Scandinavian Journal of Clinical and Laboratory Investigation. 75:3; 247-253.

35) Urbina A, Palomino F. Reticulcyte count in red blood cell units stored in AS-1. Vox Sang. 2013 May; 104(4); 331-6.

36) Lippi G, Salvagno GL, Solero GP, Franchini M, Guidi GC. Stability of blood cell counts, hematologic parameters and reticulocytes indexes on the Advia A120 hematologic analyzer. J Lab Clin Med. 2005; Dec; 146(6):333-40.

37) Mustafa, I., Al Marwani, A., Mamdouh Nasr, K., Abdulla Kano, N., Hadwan, T. Time dependent assessment of morphological changes: leuko-depleted packed red blood cells stored in SAGM. BioMed research international, 2016. Park, H. et al. Alterations in cell surface area and deformability of individual human red blood cells in stored blood. ArXiv preprint arXiv: 1506.05259,2015. 